\title{
Medición volumétrica de grasa visceral abdominal con RM y su relación con elastografía hepática en una población diabética
}

Drs. Cristóbal Serrano $\mathrm{G}^{(1,2)}$, Francisco Barrera $M^{(3)}$, Carlos Riquelme ${ }^{(4)}$, Hugo Vidal A(4), Enf. Pilar Labbé(3), Drs. Francisco Cruz $\mathrm{O}^{(4)}$, Marco Arrese $\mathrm{J}^{(3)}$, Pablo Irarrázaval $\mathbf{M}^{(2,5)}$, Cristián Tejos $\mathbf{N}^{(2,5)}$, Sergio Uribe $A^{(2,4)}$.

1. Facultad de Medicina. Pontificia Universidad Católica de Chile.

2. Centro de Imágenes Biomédicas. Pontificia Universidad Católica de Chile.

3. Departamento de Gastroenterología. Pontificia Universidad Católica de Chile.

4. Departamento de Radiología. Pontificia Universidad Católica de Chile.

5. Departamento de Ingeniería Eléctrica. Pontificia Universidad Católica de Chile.

Volumetric measurement of abdominal visceral fat by MRI and its relation to transient elastography in a diabetic population

Abstract: Background. Increase in visceral fat is associated to the development of fatty liver and liver fibrosis. Hepatic elastography is a novel noninvasive method for assessing liver fibrosis.

Objective. To evaluate the relationship between visceral adipose tissue volume (VAT), subcutaneous adipose tissue volume (SAT) as measured by Magnetic Resonance Imaging (MRI) and transient elastography (TrE) values using ARFI (Acoustic Radiation Force Impulse) in type 2 Diabetic Mellitus patients (DM2). Methods. We included 20 DM2 patients (mean age: 62 years, range: 55-75, mean weight: $77.8 \mathrm{~kg}$, range: 61.5-97). Patients underwent an MRI study in a Philips Intera 1.5T scanner. MR imaging protocol included a spectral excitation sequence centered on the fat peak. The sequence included 32 cross sections, $7 \mathrm{~mm}$ thick, from the diaphragmatic cupula to the inferior border of the kidney. VAT was measured by using the semiautomatic Image J software. Each patient underwent a hepatic elastograpy (HE); 10 ARFI measurements were performed in the right hepatic lobe. Finally, a statistical analysis was performed by applying Pearson correlation between abdominal fat volumes and ARFI scores

Results. Mean VAT was $2472 \pm 861 \mathrm{cc}$, (1173-4020 cc), whilst the mean ARFI was $1.62 \pm 0.8 \mathrm{~m} / \mathrm{s},(0.8$ $3.4 \mathrm{~m} / \mathrm{s}$ ). Correlations obtained were $r=0.08$ between VAT and ARFI ( $p=0.72) ; 0.13$ between SAT and $A R F I(p=0.57)$, and -0.06 between (VAT+SAT) and ARFI $(p=0.77)$. By subdividing the sample universe, we observed that the group with ARFI scores greater than $1.6 \mathrm{~m} / \mathrm{s}$ (7 patients) had a correlation of 0.63 between VAT and ARFI ( $p=0.12)$; of 0.66 between SAT and ARFI $(p=0.10)$, and of 0.94 between VAT+SAT and $A R F I(p=0.001)$. In the subgroup with $A R F I$ values inferior to $1.6 \mathrm{~m} / \mathrm{s}$ (13 patients), the correlation was of 0.11 between VAT and ARFI ( $p=0.71)$; of 0.26 between SAT and ARFI $(p=0.38)$, and of 0.32 between ( VAT+SAT) and ARFI ( $p=0.28)$. When adjusted for gender, ARFI scores greater than $1.6 \mathrm{~m} / \mathrm{s}$ (6 patients) in the subgroup of female patients revealed a correlation of $0.79(p=0.05)$ between ARFI and VAT, and of $0.92(p=0.009)$ for VAT+SAT. Conclusions. In DM2 patients, ARFI values greater than $1.6 \mathrm{~m} / \mathrm{s}$ seem to be indicative of a good correlation between VAT and VAT+SAT, thus indicating that those with greater liver damage may present increased abdominal fat accumulation.

Keywords: Acoustic radiation force impulse, Diabetes Mellitus type 2, Fat assessment, Intra-abdominal fat, Magnetic resonance imaging, Nonalcoholic fatty liver disease, Transient elastography, Visceral adipose tissue.

Resumen: Introducción. La acumulación de grasa visceral se asocia al desarrollo de enfermedad hepática. La elastografía hepática es un método novedoso no invasivo para evaluar fibrosis hepática.

Objetivo. Evaluar la relación entre el volumen de tejido adiposo visceral (VAT), volumen de tejido adiposo subcutáneo (SAT) medido por resonancia magnética (RM), con índices de elastografía hepática (EH) utilizando ARFI (fuerza de impulso de radiación acústica) en pacientes con Diabetes Mellitus tipo 2 (DM2). Métodos. Fueron incluidos 20 pacientes (edad promedio: 62 años, rango: 55-75 años, peso promedio: $77,8 \mathrm{~kg}$, rango: 61,5-97 kg) con DM2. Los pacientes se sometieron a un examen de $R M$ en un resonador 
Philips Intera 1.5T. Al protocolo de RM se agregó una secuencia de excitación espectral centrada en el peak de grasa. La secuencia incluyó 32 cortes transversales, grosor $7 \mathrm{~mm}$, desde la cúpula diafragmática hasta el borde inferior renal. En las imágenes se midió VAT utilizando el software Image J (freeware). En cada paciente se realizó una $E H$, utilizando ARFI con 10 medidas en lóbulo hepático derecho. Finalmente, se realizó un análisis estadístico a través de la correlación de Pearson entre los volúmenes de grasa abdominal y ARFI. Resultados. El promedio de VAT fue $2472 \pm 861 \mathrm{cc}$, (1173-4020 cc), el promedio de ARFI fue $1,62 \pm 0,8 \mathrm{~m} / \mathrm{s},(0,8-3,4 \mathrm{~m} / \mathrm{s})$. Se obtuvieron correlaciones de $r=-0,08$ entre VAT y ARFI $(p=0,72)$, de 0,13 entre $S A T$ y ARFI $(p=0,57)$, y de -0,06 entre (VAT+SAT) con ARFI $(p=0,77)$. Subdividiendo el universo muestral, se encontró que el grupo con ARFI mayor que 1,6 m/s (7 pacientes) obtuvo una correlación de 0,63 entre VAT y ARFI $(p=0,12)$, de 0,66 entre SAT y ARFI $(p=0,10)$, y de 0,94 entre (VAT+SAT) con ARFI $(p=0,001)$. En el subgrupo con ARFI inferior a $1,6 \mathrm{~m} / \mathrm{s}$ (13 pacientes) la correlación fue 0,11 entre VAT y $A R F I(p=0,71)$, de 0,26 entre SAT y ARFI $(p=0,38)$, y de 0,32 entre $(V A T+S A T)$ y ARFI $(p=0,28)$. Al ajustar por género en el subgrupo de mujeres con ARFI mayor que 1,6 m/s (6 pacientes), se encontró que la correlación de ARFI y VAT fue de 0,79 $(p=0,05)$, y de 0,92 $(p=0,009)$ para VAT+SAT.

Conclusiones. En los pacientes DM2 con índices ARFI superior a 1,6 m/s habría una buena correlación con VAT y VAT+ SAT, indicando que aquellos con mayor daño hepático tendrían más acumulación de grasa abdominal.

Palabras clave: Cuantificación de grasa, Elastografía hepática, Esteatosis hepática, Diabetes Mellitus tipo 2, Fuerza de impulso de radiación acústica, Grasa intra-abdominal, Hígado graso no alcohólico, Tejido adiposo visceral, Resonancia magnética.

Serrano $C$ y cols. Medición volumétrica de grasa visceral abdominal con RM y su relación con elastografía hepática en una población diabética. Rev Chil Radiol 2011; 17(4): 183-191.

Correspondencia: Dr. Sergio Uribe A. / suribe@med.puc.cl

Trabajo financiado a través de proyectos: Anillo ACT79 y Fondecyt 11100427, 1100864

Trabajo premiado en el Primer Encuentro Franco-Chileno de Radiología 2011, efectuado en Chile por la Sociedad Chilena de Radiología.

\section{Introducción}

La obesidad es un factor de riesgo importante en numerosas patologías, tales como resistencia insulínica, síndrome metabólico, diabetes mellitus tipo 2 (DM2), hipertensión arterial, dislipidemia, enfermedad cardiovascular y enfermedad hepática por hígado graso, entre otras ${ }^{(1-5)}$.

Para evaluar los riesgos de la obesidad, se hace énfasis en la distribución que presenta la grasa abdominal, la que se clasifica en grasa subcutánea (SAT) o grasa visceral (VAT). Esta última es la de mayor relevancia ${ }^{(6-9)}$, ya que es más activa metabólicamente y tiene mayor incidencia en el aumento de producción de adipoquinas, actividad proinflamatoria e insulinorresistencia ${ }^{(10-11)}$.

VAT es un factor de riesgo presente en numerosas enfermedades metabólicas e inflamatorias. Por ejemplo, el hígado graso no alcohólico (NAFLD), condición que predispone al desarrollo de esteatohepatitis no alcohólica (NASH), fibrosis hepática, cirrosis hepática, y hepatocarcinoma ${ }^{(12)}$.

En pacientes con DM2, se ha reportado que la presencia de hígado graso como condición inicial podría conllevar a esteatohepatitis y cirrosis en un subgrupo de pacientes ${ }^{(13-14)}$. Además, estudios en población general, muestran que la presencia de VAT podría tener un rol en la patogénesis de NAFLD, así como en el desarrollo de esteatohepatitis y fibrosis hepática ${ }^{(15-17)}$.

La antropometría es ampliamente usada como método clínico para la evaluación de la obesidad(18-19). Sin embargo, hay evidencia de que la antropometría clásica no es un buen predictor de la cantidad de VAT ${ }^{(20-21)}$. Desde hace algunos años, se han utilizado métodos más precisos para cuantificar la grasa intra abdominal basados en imágenes. Dentro de éstos, la resonancia magnética $(\mathrm{RM})^{(22-23)}$ ha ganado más aceptación, dado que no tiene efectos adversos, y no utiliza radiación ionizante ${ }^{(24)}$.

A pesar de que el "gold standard" para cuantificar grasa intra abdominal es la espectroscopía por $\mathrm{RM}$, la cuantificación de VAT y SAT se realiza habitualmente con secuencias de $\mathrm{RM}$ ponderadas en $\mathrm{T} 1^{(25)}$. Esto se debe a que la espectroscopía sólo permite mediciones locales (pocos voxeles). La cuantificación con alta resolución espacial de grandes volúmenes no es factible debido a la lentitud de la técnica ${ }^{(25)}$. Por otra parte, las imágenes de RM ponderadas en T1 pueden producir sobre estimaciones en la cuantificación de los volúmenes de grasa ${ }^{(26)}$.

Se han desarrollado técnicas alternativas de RM para cuantificar de manera más precisa volúmenes de grasa. Entre ellas están: secuencias por excitación espectral de grasa, el método DIXON ${ }^{(27-28)}$, y el método IDEAL (Iterative Decomposition with Echo Asymetry and Least squares) ${ }^{(29-30)}$.

Si bien los métodos de imágenes son muy útiles para evaluar el hígado, el gold standard para su evaluación es la biopsia hepática. Sin embargo, éste es un procedimiento invasivo, con riesgos para el 
paciente, susceptible a errores durante la toma de muestras y cuyos resultados presentan variabilidad inter e intra observador(31-32). Debido a esto, se han buscado métodos alternativos menos invasivos para evaluar de manera más precisa el grado de fibrosis hepática. Entre ellos se encuentra la elastografía hepática por ultrasonido (Transient elastography, TrE) y la tecnología ARFI (fuerza de impulso de radiación acústica).

El método TrE permite estimar la rigidez hepáti$\mathrm{ca}^{(33-34)}$, y diagnosticar fibrosis significativa y cirrosis $^{(35)}$. Incluso hay estudios que han demostrado que es factible identificar etapas tempranas de fibrosis(36). El método utiliza un principio básico de la física: las ondas de presión se propagan más rápido mientras mayor sea la rigidez del medio en el que se desplazan. Luego, TrE consiste en producir una excitación mecánica externa (una onda) en el órgano de interés (e.g el hígado) a través de la piel y medir, utilizando ultrasonido, la velocidad de propagación de la onda, con el resultado final en kilo pascales. Finalmente, es necesario evaluar las ecuaciones que relacionan velocidad y elasticidad.

En la técnica ARFI, a diferencia de TrE, la excitación mecánica del tejido es reemplazada por una excitación a través de pulsos acústicos de corta duración y alta intensidad desde el mismo transductor de ultrasonido convencional, generando una excitación mecánica desde el órgano de interés, que transmite de vuelta la velocidad de propagación de la onda ${ }^{(37-38)}$. Esta técnica también ha sido usada exitosamente para el diagnóstico de fibrosis y cirrosis hepática ${ }^{(39-41)}$.

A conocimiento de los autores no hay estudios que comparen ARFI con otros métodos diagnósticos por imagen además de TrE. Dado que detectar fibrosis hepática en sus primeros estadios es relevante y que además VAT tiene correlación con alteraciones hepáticas, es importante evaluar la relación que hay entre VAT y los resultados de ARFI.

El objetivo del presente trabajo, es verificar cuál es el grado de correlación entre la cuantificación volumétrica de grasa intra abdominal (VAT y SAT) usando RM y los resultados de la elastografía hepática medidas con ARFI en pacientes con DM2.

\section{Materiales y métodos Sujetos}

Un total de 20 pacientes (8 hombres, 12 mujeres, edad promedio: 62 años, rango: 55-75 años, peso promedio: $77,8 \mathrm{~kg}$, rango $61,5-97$ ), se sometieron a una RM de abdomen. Como criterios de inclusión se consideraron: tener diagnóstico de DM2 según criterios ADA (cumplir al menos uno de los siguientes indicadores: (i) $\mathrm{HbA} 1 \mathrm{C}>6,5 \%$, (ii) glicemia de ayuno $>126 \mathrm{mg} / \mathrm{dL}$, (iii) test de tolerancia a $75 \mathrm{~g}$ de glucosa con glicemia $>200 \mathrm{mg} / \mathrm{dL}$ a las 2 hrs post carga, (iv) glicemia > $200 \mathrm{mg} / \mathrm{dL}$ con síntomas de hiperglicemia, (i.e. polidipsia, polifagia, poliurea, pérdida de peso), edad mayor a 55 años y control regular con médico. Se consideraron los siguientes criterios de exclusión: tener enfermedad hepática conocida, excepto NAFLD, pruebas hepáticas alteradas (transaminasas mayores que 3 veces valor normal (>159 UI/L), FA y GGT mayores que 2 veces valor normal (>199 UI/L $y>99 \mathrm{UI} / \mathrm{L}$ respectivamente)), consumo de alcohol mayor a $30 \mathrm{gr} /$ día en hombres y $20 \mathrm{gr} /$ día en mujeres) y contraindicación de realizarse una resonancia magnética. El protocolo fue aprobado por el comité de ética y se obtuvo consentimiento informado de todos los pacientes.

\section{Adquisición de imágenes}

Los pacientes se sometieron a un examen de RM en un resonador clínico Philips Intera 1.5T. El protocolo incluyó distintas secuencias diagnósticas y además se agregó una secuencia espectral con excitación de grasa selectiva con los siguientes parámetros: TR $1400 \mathrm{~ms}$, TE $101 \mathrm{~ms}$, flip angle $90^{\circ}$, NSA 1, grosor de corte $7 \mathrm{~mm}$, FOV de 480 x $384 \mathrm{~mm}$ y matriz de 256 x 256 pixeles.

Un total de 32 cortes transversales, sin gap, fueron adquiridos en cada paciente, cubriendo en promedio desde la cúpula del diafragma hasta el borde inferior renal, teniendo las vértebras L4-L5 como referencia anatómica. Para reducir artefactos de movimiento, todo el volumen fue adquirido en una apnea que duró aproximadamente 18 segundos.

\section{Segmentación}

Las imágenes adquiridas fueron analizadas usando el software Image $\mathrm{J}$ (National Institute of Health, Bethesda, MD) (http://rsbweb.nih.gov/ij/) por un observador, el cual ejecutó los siguientes pasos para segmentar el VAT y SAT:

1. Transformar cada imagen de RM (Figura 1a) a una imagen binaria (blanco o negro, Figura 1b), siendo blanco los pixeles de grasa y negro los que no contienen grasa. La binarización se realizó comparando los niveles de gris de la imagen original contra un valor umbral escogido heurísticamente para cada paciente.

2. Los artefactos presentes en las imágenes producto de inhomogeneidades de campo o susceptibilidades magnéticas fueron corregidos manualmente. Esto se realizó comparando las imágenes originales con los cortes superiores e inferiores de cada imagen. Un ejemplo de un área afectada por artefactos y el resultado de la corrección se indican con flechas en las Figuras $1 a$ y $1 b$.

3. Se cuantificó el volumen total de grasa abdominal (VAT+SAT) como el número de pixeles blancos de todas las imágenes binarias, multiplicando por el volumen de cada pixel (i.e. el espesor de 
corte por el área de un pixel).

4. Para separar VAT y SAT se delineó (en forma semi-automática y en cada una de las imágenes binarias) el contorno interno de la cavidad visceral. De esta forma se generó un área delimitada para medir el volumen de VAT (Figura 1c).

5. Se cuantificó el volumen total de VAT de igual forma que en el paso 3, pero considerando sólo el área interna de los contornos definidos en el paso 4.

6. Para medir SAT, se restó el volumen de VAT al total de la grasa abdominal (VAT+SAT). Esto equivale a hacer los cálculos de volumen excluyendo la región de VAT en cada corte (Figura 1d).

El proceso de análisis de las imágenes duró aproximadamente 50 minutos por paciente.

\section{Ultrasonido y Elastrografía Hepática ARFI}

Veinte pacientes con sospecha de daño hepático crónico (DHC) fueron sometidos a una EH utilizando ARFI en equipo Siemens S8000. Dos operadores independientes, realizaron 10 mediciones en el lóbulo hepático derecho de cada paciente. Las mediciones se realizaron en apnea, evitando inspiración y espiración extremas. Basado en los criterios de Nguyen et al ${ }^{(42)}$, se definió como ausencia de fibrosis significativa los valores obtenidos hasta 1,28 m/s $(6 \mathrm{kPa})$; fibrosis (F2) entre $1,29 \mathrm{~m} / \mathrm{s}$ y $1,63 \mathrm{~m} / \mathrm{s}(6-12 \mathrm{kPa}), \mathrm{y}$ fibrosis avanzada o cirrosis (F3-F4) mayor a 1,64 m/s $(12 \mathrm{kPa})$. La transformación entre elasticidad $(\mathrm{kPa})$ y velocidad $(\mathrm{m} / \mathrm{s})$ se realizó de la siguiente manera:

Velocidad $(\mathrm{m} / \mathrm{s})=0,92+[0,06 \times$ elasticidad $(\mathrm{kPa})]$

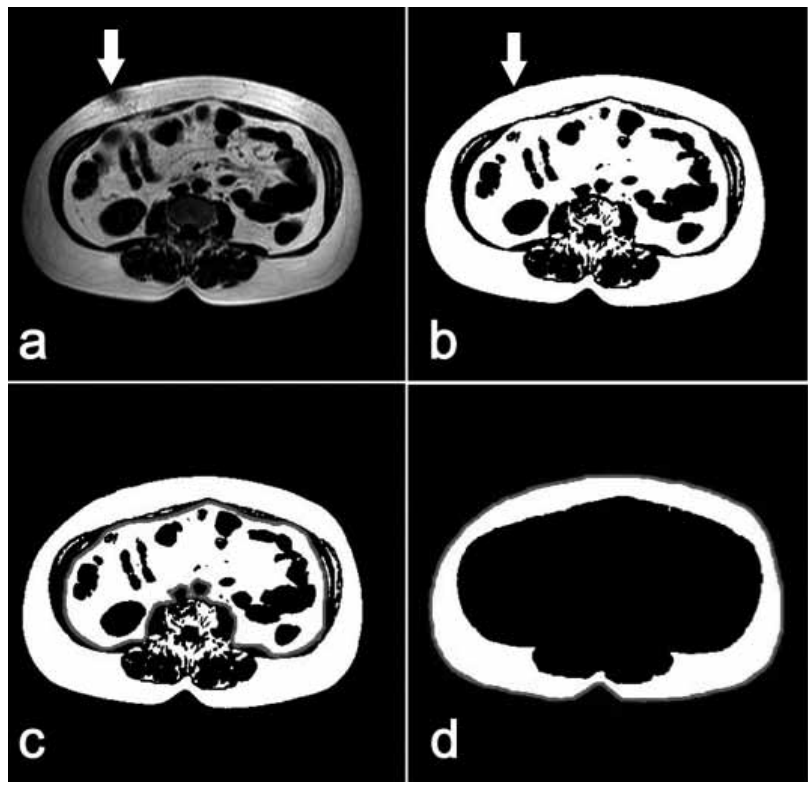

186

\section{Método estadístico}

Promedios y desviaciones estándar fueron usados para describir la muestra en estudio. En cada paciente, se calculó la correlación de Pearson (r), entre SAT, VAT, y VAT+SAT asociado a cada variable elastográfica.

Se estableció $P<0,05$ para indicar significancia estadística de la hipótesis evaluada: la existencia de alguna asociación entre el volumen de grasa (medido en las imágenes de RM) y la medición de elastografía hepática por ARFI. Todos los análisis fueron realizados usando el software Minitab 15.

\section{Resultados}

En la Tabla I se muestra un resumen descriptivo de todas las variables en estudio.

De los 20 pacientes, 12 son mujeres (IMC promedio: $30,59,50 \%$ catalogadas como obesas) y 8 hombres (IMC promedio: 27,57, 12,5\% catalogados como obesos).

Los volúmenes promedio (medidos a partir de las imágenes de RM) fueron: VAT $2472 \pm 861 \mathrm{cc}$ (rango: 1173-4020 cc), SAT $3093 \pm 1293 \mathrm{cc}$ (rango: $1161-5617 \mathrm{cc}$ ) y para VAT+SAT $5565 \pm 1405 \mathrm{cc}$ (rango: 3413-8129 cc).

La Figura 2 muestra un gráfico por dispersión entre las medidas de grasa por RM y velocidad de propagación medido con ARFI, donde se observa en general que no hay una buena correlación. Sin embargo, en pacientes con velocidad de propagación superior a 1,6m/s (datos destacados en azul), hay una mejor correlación con la cantidad de grasa, en especial con VAT+SAT.

En la Tabla II y III se muestra un resumen descriptivo de todas las variables ajustadas por velocidades de propagación menores y superiores a $1,6 \mathrm{~m} / \mathrm{s}$ medidas por ARFI. Ambos subgrupos presentan IMC promedio similar (29,4 y 29,3 respectivamente).

Los resultados de la correlación de Pearson entre VAT, SAT y VAT+SAT y la velocidad de propagación medida por ARFI se muestran en la Tabla IV.

Al correlacionar los resultados de las mediciones de grasa con los resultados de todas las velocidades, se encontró una baja correlación $(r<0,1)$, similares resultados se encontraron para aquellos con velocidades menor a $1,6 \mathrm{~m} / \mathrm{s}$.

Sin embargo, para el subgrupo con velocidades superior a $1,6 \mathrm{~m} / \mathrm{s}$ se apreciaron correlaciones mayores, destacándose una gran correlación con la grasa total (VAT+SAT) $(r=0,94, p<0,01)$. Resultados similares se encontraron al analizar las distintas correlaciones por género. En la cual se observa en el grupo mujeres, correlación entre VAT y velocidades mayores a 1,6 $\mathrm{m} / \mathrm{s}(\mathrm{r}=0,79, \mathrm{p}<0,05)$.

Figura 1. Imagen de RM original (a) e imagen binaria (b) tejido adiposo visceral (c) y tejido adiposo subcutáneo (d). Las flechas en (a) y (b) indican un artefacto en la imagen y su correlación para el cálculo de volumen de grasa, respectivamente. 
Tabla I. Estadística descriptiva de cada variable en estudio, $n=20$ (Minitab).

\begin{tabular}{lccc|} 
Variable & Media & Desviación estándar & Rango \\
\hline VAT (cc) & 2472 & 861 & $1173-4020$ \\
SAT (cc) & 3093 & 1283 & $1161-5617$ \\
VAT+SAT (cc) & 5565 & 1405 & $3413-8129$ \\
Velocidad ARFI (m/s) & 1,62 & 0,792 & $0,88-3,46$ \\
Edad (años) & 61,80 & 5,02 & $55-75$ \\
Peso (kg) & 77,80 & 11,17 & $61,5-97$ \\
Talla (m) & 1,63 & 0,09 & $1,42-1,81$ \\
IMC (kg/m²) & 29,39 & 4,58 & $22,6-37,4$ \\
(VAT): tejido adiposo visceral. (SAT): tejido adiposo subcutáneo. (ARFI): fuerza de impulso de radiación acústica. \\
(IMC): índice masa corporal.
\end{tabular}

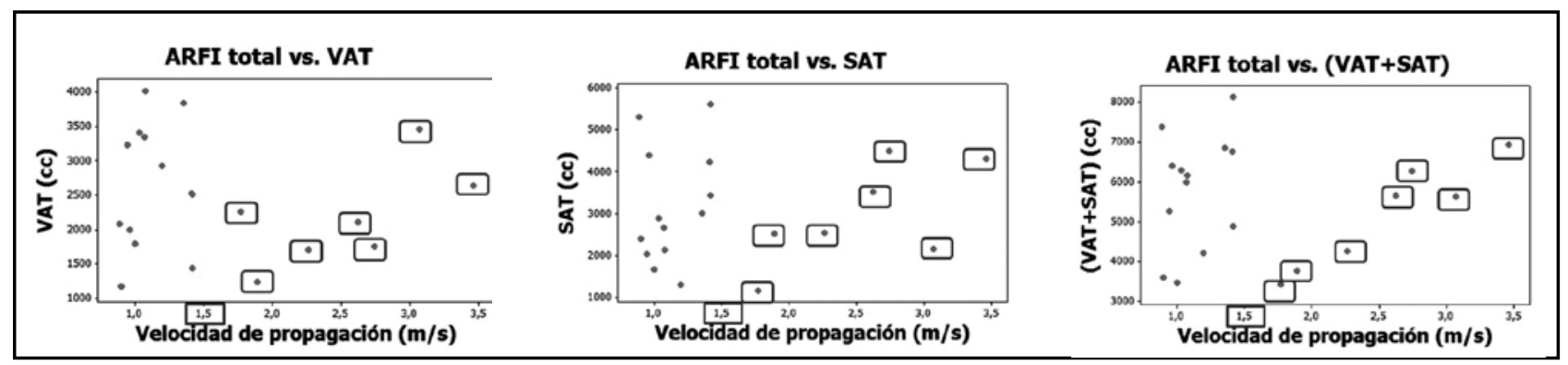

Figura 2. Gráfica de dispersión entre la cantidad de grasa (VAT y/o SAT) con todas las velocidades y velocidades $\geq 1,6 \mathrm{~m} / \mathrm{s}$, medidas por ARFI (Minitab).

Tabla II. Estadística descriptiva para variables con ARFI $\geq 1,6 \mathrm{~m} / \mathrm{s}$.

\begin{tabular}{lccc|} 
Variable & Media & Desviación estándar & Rango \\
\hline VAT (cc) & 2166 & 724 & $1239-3463$ \\
SAT (cc) & 2962 & 1210 & $1161-4507$ \\
VAT+SAT (cc) & 5128 & 1335 & $3413-6944$ \\
Velocidad ARFI (m/s) & 2,54 & 0,61 & $1,77-3,46$ \\
Edad (años) & 62,20 & 4,27 & $57-68$ \\
Peso (kg) & 75,00 & 9,94 & $61,5-87$ \\
Talla (m) & 1,59 & 0,07 & $1,50-1,73$ \\
IMC (kg/m²) & 29,40 & 4,54 & $23,1-34,5$ \\
(VAT): tejido adiposo visceral. (SAT): tejido adiposo subcutáneo. (ARFI): fuerza de impulso de radiación acústica. \\
(IMC): índice de masa corporal.
\end{tabular}


Tabla III. Estadística descriptiva para variables con ARFI $<1,6 \mathrm{~m} / \mathrm{s}$

\begin{tabular}{lccc|} 
Variable & Media & Desviación estándar & Rango \\
\hline VAT (cc) & 2636 & 909 & $1173-4020$ \\
SAT (cc) & 3164 & 1363 & $1298-5617$ \\
VAT+SAT (cc) & 5800 & 1436 & $3469-8129$ \\
Velocidad ARFI (m/s) & 1,12 & 0,20 & $0,88-1,41$ \\
Edad (años) & 61,50 & 5,53 & $55-75$ \\
Peso (kg) & 79,30 & 11,88 & $63-97$ \\
Talla (m) & 1,64 & 0,10 & $1,42-1,81$ \\
IMC (kg/m ${ }^{2}$ ) & 29,30 & 4,79 & $22,6-37,4$ \\
(VAT): tejido adiposo visceral. (SAT): tejido adiposo subcutáneo. (ARFI): fuerza de impulso de radiación acústica. \\
(IMC): índice de masa corporal
\end{tabular}

Tabla IV. Análisis estadístico. Correlación de Pearson (r), entre VAT, SAT y (VAT+SAT) con todas las velocidades de propagación, velocidades $\geq 1,6 \mathrm{~m} / \mathrm{s}$ y velocidades $<1,6 \mathrm{~ms}$, medidas por ARFI, ajustado por género, $n=20$. (Minitab)

\begin{tabular}{|c|c|c|c|c|c|c|c|c|c|}
\hline \multirow[t]{2}{*}{ Variables } & \multicolumn{3}{|c|}{ Todas las velocidades } & \multicolumn{3}{|c|}{ Velocidad $<1,6 \mathrm{~m} / \mathrm{s}$} & \multicolumn{3}{|c|}{ Velocidad $\geq 1,6 \mathrm{~m} / \mathrm{s}$ * } \\
\hline & Total & |Mujeres & |Hombres & Total & |Mujeres & |Hombres & Total & |Mujeres & Homb \\
\hline VAT & $\begin{array}{l}r=-0,08 \\
p=0,72\end{array}$ & $\begin{array}{l}r=0,45 \\
p=0,13\end{array}$ & $\begin{array}{l}r=-0,23 \\
p=0,57\end{array}$ & $\begin{array}{l}r=0,11 \\
p=0,71\end{array}$ & $\begin{array}{l}r=0,40 \\
p=0,42\end{array}$ & & $\begin{array}{l}r=0,63 \\
p=0,12\end{array}$ & $\begin{array}{l}r=0,79 \\
p=0,05\end{array}$ & $\begin{array}{l}r=I D \\
p=I D\end{array}$ \\
\hline SAT & $\begin{array}{l}r=-0,13 \\
p=0,57\end{array}$ & $\begin{array}{l}r=-0,24 \\
p=0,44\end{array}$ & $\begin{array}{l}r=-0,03 \\
p=0,36\end{array}$ & $\begin{array}{l}r=0,26 \\
p=0,38\end{array}$ & $\begin{array}{l}r=0,18 \\
p=0,73\end{array}$ & $\begin{array}{l}r=0,27 \\
p=0,55\end{array}$ & $\begin{array}{l}r=0,66 \\
p=0,10\end{array}$ & $\begin{array}{l}r=0,48 \\
p=0,33\end{array}$ & $\begin{array}{l}r=I D \\
p=I D\end{array}$ \\
\hline$(\mathrm{VAT}+\mathrm{SAT})$ & $\begin{array}{l}r=-0,06 \\
p=0,77\end{array}$ & $\begin{array}{l}r=0,01 \\
p=0,96\end{array}$ & $\begin{array}{l}r=-0,33 \\
p=0,42\end{array}$ & $\begin{array}{l}r=0,32 \\
p=0,28\end{array}$ & $\begin{array}{l}r=0,26 \\
p=0,61\end{array}$ & $\begin{array}{l}r=0,37 \\
p=0,41\end{array}$ & $\begin{array}{l}r=0,94 \\
p=0,001\end{array}$ & $\begin{array}{l}r=0,92 \\
p=0,009\end{array}$ & $\begin{array}{l}r=I D \\
p=I D\end{array}$ \\
\hline
\end{tabular}

(VAT): tejido adiposo visceral. (SAT): tejido adiposo subcutáneo. (ARFI): fuerza de impulso de radiación acústica. (IMC): índice de masa corporal. *: ARFI $\geq 1,6 \mathrm{~m} / \mathrm{s}$, sólo había 1 hombre (total $A R F I \geq 1,6 \mathrm{~m} / \mathrm{s}, \mathrm{n}=7$ ).

ID: Insuficientes datos para analizar.

\section{Discusión}

Es sabido que pacientes con DM2 tienen más riesgo de tener enfermedades derivadas de la grasa metabólicamente activa, como lo es la esteatosis hepática y las enfermedades cardiovasculares ${ }^{(15)}$. Por otra parte, los sujetos diabéticos evolucionan con mayor frecuencia con fibrosis hepática incluso con transaminasas normales ${ }^{(43)}$. Por ello, es importante investigar la magnitud del daño hepático mediante métodos no invasivos como ARFI. Por otra parte, estudios recientes sugieren que la fibrogénesis hepática es un proceso dinámico y al menos parcialmente reversible ${ }^{(31)}$, por lo cual, es clave detectar las etapas iniciales de esta enfermedad.

Uno de los factores que se ha asociado directamente en forma importante a la magnitud de fibrosis en pacientes con NAFLD es el VAT ${ }^{(16)}$. Resulta trascendente determinar la relación entre VAT y la magnitud de fibrosis hepática, y establecer su utilidad como parámetro para vigilancia de sujetos diabéti- 
cos con alto riesgo de desarrollo de complicaciones hepáticas severas.

Nuestros resultados indican que al analizar una población con $\mathrm{DM} 2$, las velocidades de propagación (o rigidez) en el hígado medidas por ARFI no tendrían buena correlación con la cantidad de VAT medida por RM. Sin embargo, al separar el grupo de pacientes que tienen velocidades de propagación sobre $1,6 \mathrm{~m} / \mathrm{s}$, se puede establecer una buena correlación con VAT $(r=0,79, p<0,05)$, y con la cantidad de grasa total (VAT+SAT) $(r=0,9, p=<0,05)$. Sin embargo, es importante extender esta investigación en una muestra más grande de pacientes.

Recientes estudios han demostrado que la elastografía hepática por ultrasonido, puede realizar un diagnóstico adecuado de fibrosis hepática(33-36). No obstante, esta técnica tiene algunas limitaciones. En pacientes con obesidad severa (IMC $>40 \mathrm{~kg}$ / $\mathrm{m}^{2}$ ), la intensidad de las ondas acústicas se podrían ver atenuadas, comprometiendo la precisión de las medidas de velocidad( ${ }^{(31)}$. Se ha discutido si la obesidad pudiera alterar significativamente la medición de rigidez hepática ${ }^{(44)}$. En cambio, en relación a la elastografía medida por ARFI existen algunos reportes que sugieren que se lograría una estimación adecuada de fibrosis hepática incluso en sujetos obesos ${ }^{(45)}$.

Se ha visto que ARFI es muy sensible para detectar ausencia de fibrosis hepática significativa (velocidades menores a $1,29 \mathrm{~m} / \mathrm{s}$ ) y presencia de fibrosis severa 0 cirrosis (velocidades mayores a 1,64 m/s). Sin embargo, hay una zona gris (velocidades entre $1,29 \mathrm{~m} / \mathrm{s}$ y $1,64 \mathrm{~m} / \mathrm{s}$ ) donde ARFI no puede diferenciar el grado de fibrosis hepática en forma precisa ${ }^{(42)}$. Además se ha estipulado, que a mayor valor de elastografía, aumenta la probabilidad de tener fibrosis avanzada. En nuestro estudio, encontramos que en aquellos pacientes que tienen una velocidad de propagación superior a $1,6 \mathrm{~m} / \mathrm{s}$, hay una asociación directa con la cantidad de grasa abdominal y VAT. Por ello, medir correctamente la cantidad de grasa en este tipo de pacientes también podría ayudar en el diagnóstico de esta enfermedad. Un aspecto relevante a evaluar en el futuro es si aquellos sujetos con valores elevados de elastografía asociados a altos valores de VAT y/o VAT+SAT se asocian a mayor grado de fibrosis en la biopsia hepática. De esta manera, poder agrupar marcadores basados en imágenes, indirectos y no invasivos, que se correlacionen bien con avanzada fibrosis hepática, sin necesidad de recurrir a una biopsia hepática.

En este trabajo la medición de grasa se realizó por RM utilizando una secuencia de excitación espectral de grasa. Se prefirió usar un método de imágenes dado que hay reportes que indican que las medidas antropométricas usadas en la clínica hoy en día no son un buen indicador de la cantidad de grasa abdominal(20-21).
La ventaja de usar RM con excitación selectiva, es que sólo obtenemos señal de la grasa y por lo tanto es fácil segmentar la grasa VAT y SAT. Sin embargo, esta secuencia presenta problemas debido a su sensibilidad a las inhomogeneidades de campo $^{(46,47)}$.

Para solucionar este problema se han desarrollado recientemente otros métodos. Por ejemplo, el método IDEAL, el cual permite obtener imágenes de agua y grasa simultáneamente, corrigiendo las inhomogeneidades de campo y decaimiento de señal debido a T2*(29,46). Sin embargo, esta secuencia no está disponible aún para equipos de RM estándares.

En conclusión, en pacientes DM2 con velocidades de propagación de onda hepática medidas por ARFI superiores a $1,6 \mathrm{~m} / \mathrm{s}$, hay una alta correlación positiva estadísticamente significativa entre la cantidad de VAT (y/o VAT+SAT), y un mayor riesgo de fibrosis. Sin embargo, es necesario investigar si estos resultados se asocian a mayor grado de fibrosis en biopsia hepática e investigar cuál es el origen de esta relación.

\section{Bibliografía}

1. Mann GV. The influence of obesity in health. [Review Part 1] N Engl J Med 1974; 291: 178-185. [Review Part 2: 1974; 291: 226-232].

2. Wilson PW, D'Agostino RB, Sullivan L, Parise H, Kannel WB. Overweight and obesity as determinants of cardiovascular risk: the Framingham experience. Arch Intern Med 2002; 162: 1867-1872.

3. Manson JE, Colditz GA, Stampfer MJ, et al. A prospective study of obesity and risk of coronary heart disease in women. N Engl J Med 1990; 322: 882-889.

4. Kenchaiah S, Evans JC, Levy D, et al. Obesity and the risk of heart failure. N Engl J Med 2002; 347: 305-313.

5. Sharma AM, Chetty VT. Obesity, hypertension and insulin resistance. Acta Diabetol 2005; 42(Suppl 1): S3-S8.

6. Kitabchi $A E$, Buffington CK. Body fat distribution, hyperandrogenicity, and health risks. Semin Reprod Endocrinol 1994; 12: 6-14.

7. Albu JB, Kovera AJ, Johnson JA. Fat distribution and health in obesity. Ann N Y Acad Sci 2000; 904: 491501.

8. Lapidus L, Bengtsson C, Larsson B, Pennert K, Rybo E, Sjöström L. Distribution of adipose tissue and risk of cardiovascular disease and death: a 12 year follow up of participants in the population study of women in Gothenburg, Sweden. Br Med J (Clin Res Ed). 1984; 289: 1257-1261.

9. Despres JP, Moorjani S, Ferland M, et al. Adipose tissue distribution and plasma lipoprotein levels in obese women: importance of intra-abdominal fat. Arteriosclerosis 1989; 9: 203-210.

10. Bruunsgaard $\mathrm{H}$, Pedersen BK. Age-related inflammatory cytokines and disease. Immunol Allergy 2003; 23: 1539.

11. Gastaldelli A, Sironi AM, Ciociaro D, et al. Visceral fat and beta cell function in non-diabetic humans. Diabetologia 2006; 48: 2090-2096.

12. Bugianesi E, Leone N, Vanni E, Marchesini G, Brunello $F$, Carucci P, et al. Expanding the natural history of 
non-alcoholic steatohepatitis: from cryptogenic cirrhosis to hepatocellular carcinoma. Gastroenterology 2002; 123: 134-140.

13. Williamson RM, Price JF, Glancy S, Perry E, Nee LD, Hayes PC, Frier BM, Van Look LA, Johnston Gl, Reynolds RM, Strachan MW. Prevalence of and risk factors for hepatic steatosis and nonalcoholic Fatty liver disease in people with type 2 diabetes: the edinburgh type 2 diabetes study. Diabetes Care 2011; 34(5): 1139-1144.

14. Ismail $\mathrm{MH}$. Nonalcoholic fatty liver disease and type 2 diabetes mellitus: the hidden epidemic. Am J Med Sci. 2011; 341(6): 485-492.

15. Eguchi $Y$, Mizuta T, Sumida $Y$, Ishibashi E, Kitajima $Y$, Isoda $\mathrm{H}$, Horie $\mathrm{H}$, Tashiro $\mathrm{T}$, Iwamoto $\mathrm{E}$, Takahashi $\mathrm{H}$, Kuwashiro T, Soejima S, Kawaguchi Y, Oda Y, Emura S, Iwakiri R, Ozaki I, Eguchi T, Ono N, Anzai K, Fujimoto $\mathrm{K}$, Koizumi S. The pathological role of visceral fat accumulation in steatosis, inflammation, and progression of nonalcoholic fatty liver disease. J Gastroenterol 2011; 46 (Suppl 1): 70-78. Epub 2010 Nov 2.

16. Van der Poorten D, Milner KL, Hui J, Hodge A, Trenell MI, Kench JG, London R, Peduto T, Chisholm DJ, George J. Visceral fat: a key mediator of steatohepatitis in metabolic liver disease. Hepatology 2008; 48(2): 449-457.

17. Kuk JL, Katzmarzyk PT, Nichaman MZ, Church TS, Blair SN, Ross R. Visceral fat is an independent predictor of all-cause mortality in men. Obesity (Silver Spring). 2006; 14(2): 336-341.

18. Expert Panel on the Identification, Evaluation, and Treatment of Overweight in Adults. Clinical guidelines on the identification, evaluation, and treatment of overweight and obesity in adults: executive summary. Am J Clin Nutr 1998; 68: 899-917.

19. WHO. Obesity: preventing and managing the global epidemic: report of a WHO consultation. World Health Organ Tech Rep Ser 2000; 894: 1-253.

20. Ferrannini E, Sironi A, lozzo P, Gastaldelli A. Intraabdominal adiposity, abdominal obesity, and cardiometabolic risk. Eur Heart J Suppl 2007 Eur Heart J Suppl 2008; 10: B4-B10.

21. Van der Kooy K, Leenen R, Seidell JC, Deurenberg P, Droop A, Bakker CJ. Waist-hip ratio is a poor predictor of changes in visceral fat. Am J Clin Nutr 1993; 57: 327-333.

22. Ellis KJ. Human body composition: in vivo methods. Physiol Rev 2000; 80: 649-680.

23. Seidell JC, Bakker CJ, van der Kooy K. Imaging techniques for measuring adipose-tissue distribution-a comparison between computed tomography and 1.5-T magnetic resonance. Am J Clin Nutr 1990; 51: 953-957.

24. Staten MA, Totty WG, Kohrt WM. Measurement of fat distribution by magnetic resonance imaging. Invest Radiol 1989; 24: 345-349.

25. Barac-Nieto M, Gupta RK. Use of proton MR spectroscopy and MR imaging to assess obesity. JMagn Reson Imaging 1996; 6: 235-238.

26. Dimitrios C. Karampinos, Huanzhou Yu, Ann Shimakawa, Thomas M. Link, Sharmila Majumdar. T1-Corrected Fat Quantification Using Chemical Shift-Based Water/ Fat Separation: Application to Skeletal Muscle. Magn Reson Med 2011 Mar 30. doi: 10.1002/mrm.22925.

27. Kaldoudi E, Williams SC, Barker GJ, Tofts PS. A chemical shift selective inversion recovery sequence for fat-suppressed MRI: theory and experimental validation. Magn Reson Imaging 1993; 11: 341-355.

28. Dixon WT. Simple proton spectroscopic imaging. Radiology 1984; 153: 189-194.

29. Reeder SB, McKenzie CA, Pineda AR, Yu H, Shimakawa A, Brau AC, Hargreaves BA, Gold GE, Brittain JH. Water-fat separation with IDEAL gradient-echo imaging. J Magn Reson Imaging 2007; 25: 644-652.

30. Yu H, McKenzie CA, Shimakawa A, Vu AT, Brau AC, Beatty PJ, Pineda AR, Brittain JH, Reeder SB. Multiecho reconstruction for simultaneous water-fat decomposition and T2* estimation. J Magn Reson Imaging 2007; 26(4): 1153-1161.

31. Manning DS, Afdhal NH. Diagnosis and quantitation of fibrosis. Gastroenterology. 2008; 134(6): 1670-1681.

32. Regev A, Berho M, Jeffers LJ, et al. Sampling error and intraobserver variation in liver biopsy in patients with chronic HCV infection. Am J Gastroenterol 2002; 97: 2614-2618.

33. Sandrin L, Fourquet B, Hasquenoph JM, et al. Transient elastography: a new noninvasive method for assessment of hepatic fibrosis. Ultrasound Med Biol 2003; 29: 1705-1713.

34. Rockey DC. Noninvasive assessment of liver fibrosis and portal hypertension with transient elastography. Gastroenterology 2008; 134(1): 8-14.

35. Talwalkar JA, Kurtz DM, Schoenleber SJ, et al. Ultrasound based transient elastography for the detection of hepatic fibrosis: systematic review and meta-analysis. Clin Gastroenterol Hepatol 2007; 5: 1214-1220.

36. Shaheen AA, Wan AF, Myers RP. FibroTest and FibroScan for the prediction of hepatitis C-related fibrosis: a systematic review of diagnostic test accuracy. Am J Gastroenterol 2007; 102: 2589-2600.

37. Palmeri ML, Wang MH, Dahl JJ, Frinkley KD, Nightingale KR. Quantifying hepatic shear modulus in vivo using acoustic radiation force. Ultrasound Med Biol 2008; 34: 546-558.

38. Lupsor M, Badea R, Stefanescu H, Sparchez Z, Branda $H$, Serban A, et al. Performance of a new elastographic method (ARFI technology) compared to unidimensional transient elastography in the noninvasive assessment of chronic hepatitis C. Preliminary results. J Gastrointestin Liver Dis 2009; 18: 303-310.

39. Ebinuma $H$, Saito $H$, Komuta M, Ojiro K, Wakabayashi K, Usui S, Chu PS, Umeda R, Ishibashi Y, Takayama T, Kikuchi M, Nakamoto N, Yamagishi Y, Kanai T, Ohkuma K, Sakamoto M, Hibi T. Evaluation of liver fibrosis by transient elastography using acoustic radiation force impulse: comparison with Fibroscan(®). J Gastroenterol. 2011 Jul 21.

40. Friedrich-Rust M, Wunder K, Kriener S, Sotoudeh F, Richter S, Bojunga J, Herrmann E, Poynard T, Dietrich CF, Vermehren J, Zeuzem S, Sarrazin C. Liver fibrosis in viral hepatitis: noninvasive assessment with acoustic radiation force impulse imaging versus transient elastography. Radiology. 2009; 252(2): 595-604.

41. Haque M, Robinson C, Owen D, Yoshida EM, Harris A. Comparison of acoustic radiation force impulse imaging (ARFI) to liver biopsy histologic scores in the evaluation of chronic liver disease: A pilot study. Ann Hepatol 2010; 9(3): 289-293.

42. Nguyen D, Talwalkar JA. Noninvasive assessment of liver fibrosis. Hepatology 2011; 53(6): 2107-2110. 
43. Fracanzani AL, Valenti L, Bugianesi E, Andreoletti M, Colli A, Vanni E, Bertelli C, Fatta E, Bignamini D, Marchesini G, Fargion S. Risk of severe liver disease in nonalcoholic fatty liver disease with normal aminotransferase levels: a role for insulin resistance and diabetes. Hepatology 2008; 48(3): 792-798.

44. Wong VW, Vergniol J, Wong GL, Foucher J, Chan HL, Le Bail B, Choi PC, Kowo M, Chan AW, Merrouche W, Sung JJ, de Lédinghen V. Diagnosis of fibrosis and cirrhosis using liver stiffness measurement in nonalcoholic fatty liver disease. Hepatology 2010; 51(2): 454-462.

45. J Palmeri ML, Wang MH, Rouze NC, Abdelmalek MF,
Guy CD, Moser B, Diehl AM, Nightingale KR. Noninvasive evaluation of hepatic fibrosis using acoustic radiation force-based shear stiffness in patients with nonalcoholic fatty liver disease. Hepatol 2011; 55(3): 666-672. Epub 2011 Jan 21.

46. Hu HH, Nayak KS, Goran MI. Assessment of abdominal adipose tissue and organ fat content by magnetic resonance imaging. Obes Rev 2011; 12(5): e504-515.

47. Peng Q, McColl RW, Ding Y, Wang J, Chia JM, Weatherall $\mathrm{PT}$. Automated method for accurate abdominal fat quantification on water-saturated magnetic resonance images. J Magn Reson Imaging 2007; 26: 738-746.

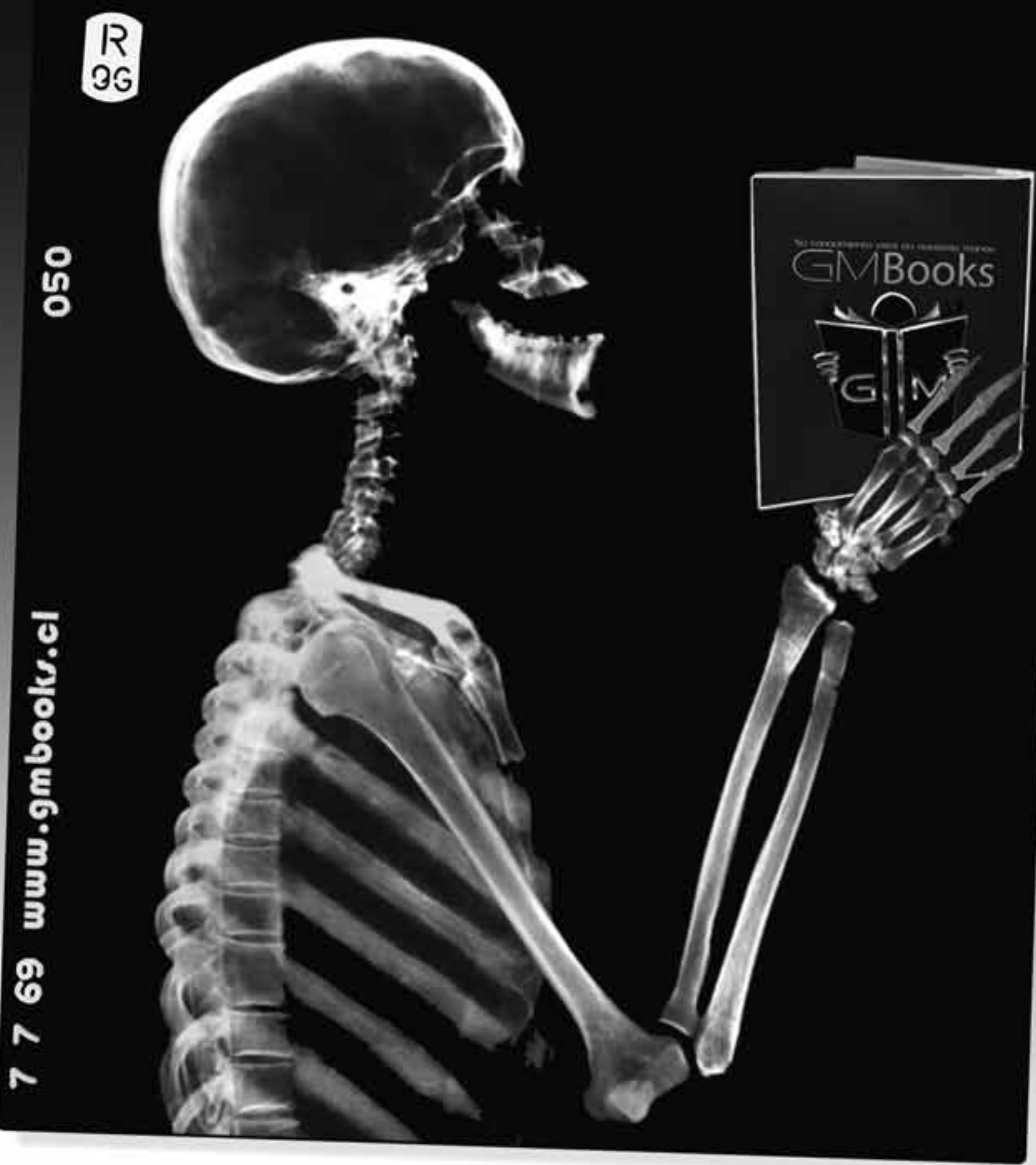

- Importación directa de libros de medicina

- Rápidez, seriedad y puntualidad de entrega de libros

- Servicio integral de calidad

- Atención personalizada

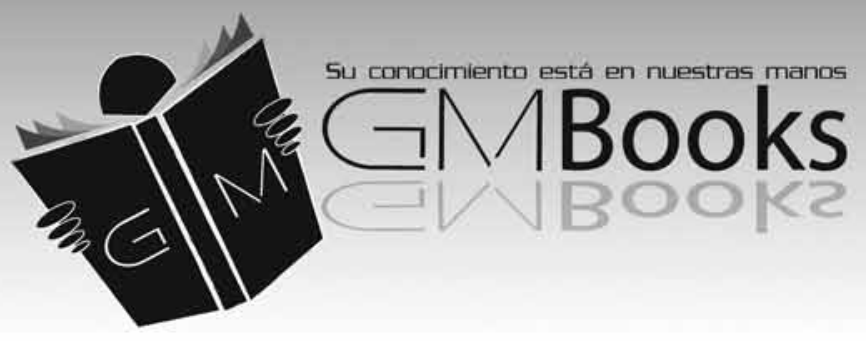

